\title{
Identifying the Critical Links in Road Transportation Networks: Centrality- based Approach Utilizing Structural Properties
}

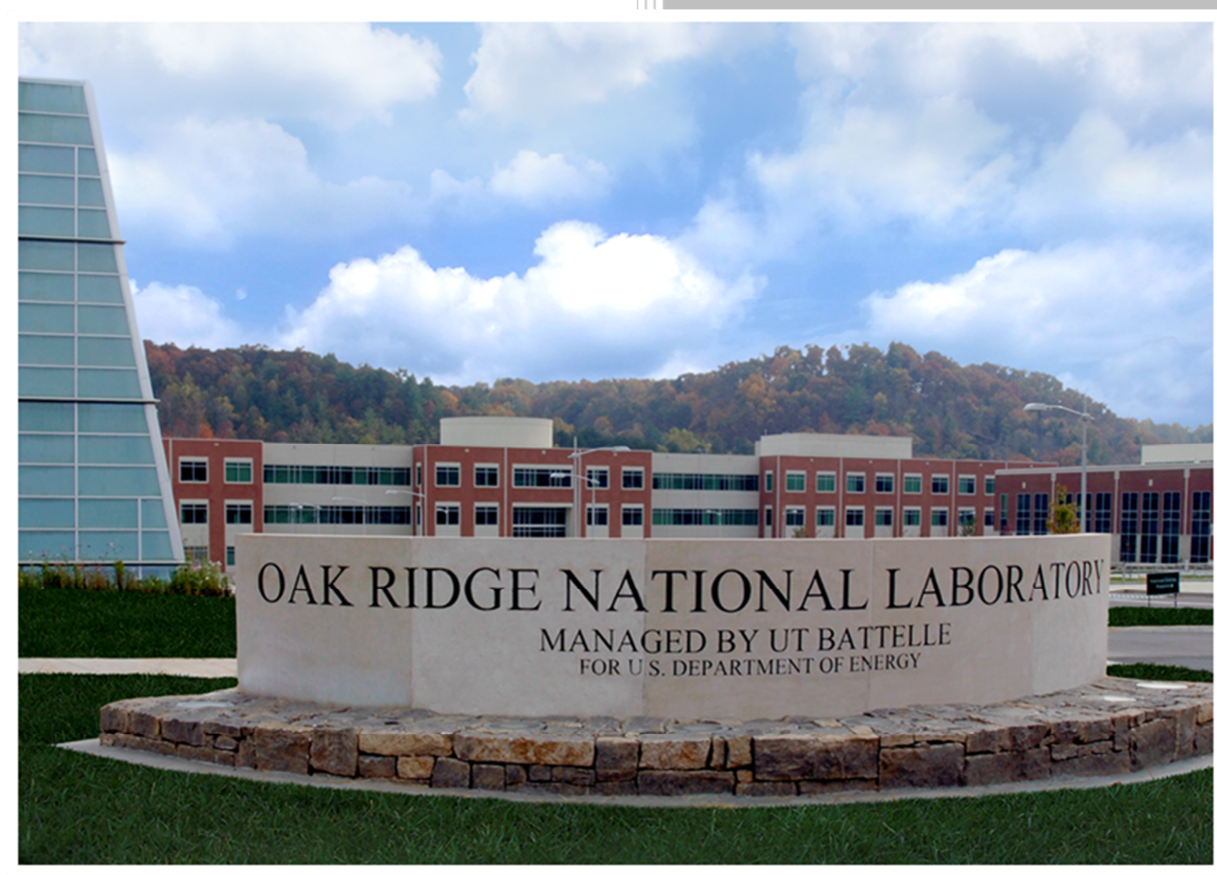

\section{Approved for public release;}

H M Abdul Aziz

Supriya Chinthavali

Sangkeun(Matt) Lee

distribution is unlimited. 


\title{
DOCUMENT AVAILABILITY
}

Reports produced after January 1, 1996, are generally available free via US Department of Energy (DOE) SciTech Connect.

Website http://www.osti.gov/scitech/

Reports produced before January 1, 1996, may be purchased by members of the public from the following source:

\author{
National Technical Information Service \\ 5285 Port Royal Road \\ Springfield, VA 22161 \\ Telephone 703-605-6000 (1-800-553-6847) \\ TDD 703-487-4639 \\ Fax 703-605-6900 \\ E-mail info@ntis.gov \\ Website http://www.ntis.gov/help/ordermethods.aspx
}

Reports are available to DOE employees, DOE contractors, Energy Technology Data Exchange representatives, and International Nuclear Information System representatives from the following source:

Office of Scientific and Technical Information

PO Box 62

Oak Ridge, TN 37831

Telephone 865-576-8401

Fax 865-576-5728

E-mail reports@osti.gov

Website http://www.osti.gov/contact.html

This report was prepared as an account of work sponsored by an agency of the United States Government. Neither the United States Government nor any agency thereof, nor any of their employees, makes any warranty, express or implied, or assumes any legal liability or responsibility for the accuracy, completeness, or usefulness of any information, apparatus, product, or process disclosed, or represents that its use would not infringe privately owned rights. Reference herein to any specific commercial product, process, or service by trade name, trademark, manufacturer, or otherwise, does not necessarily constitute or imply its endorsement, recommendation, or favoring by the United States Government or any agency thereof. The views and opinions of authors expressed herein do not necessarily state or reflect those of the United States Government or any agency thereof. 


\title{
IDENTIFYING THE CRITICAL LINKS IN ROAD TRANSPORTATION NETWORKS: CENTRALITY-BASED APPORACH UTILIZING STRUCTURAL PROPERTIES
}

\author{
H M Abdul Aziz \\ Supriya Chinthavali \\ Sangkeun(Matt) Lee
}

March 21, 2016

Prepared by

OAK RIDGE NATIONAL LABORATORY

Oak Ridge, TN 37831-6283

managed by

UT-BATTELLE, LLC

for the

US DEPARTMENT OF ENERGY

under contract DE-AC05-00OR22725 



\section{CONTENTS}

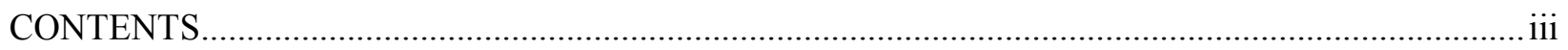

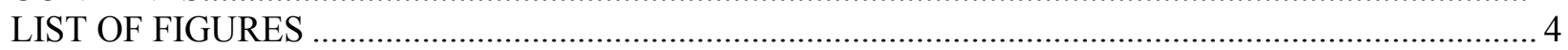

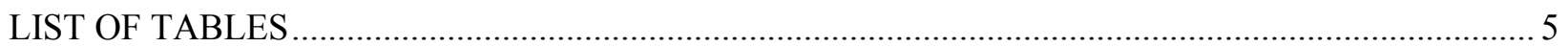

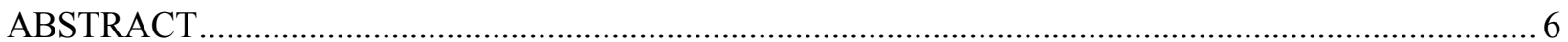

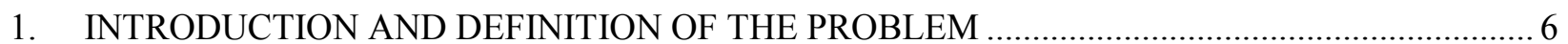

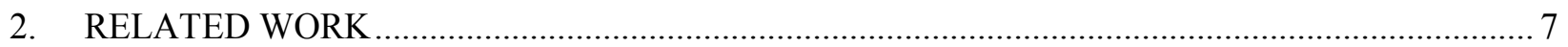

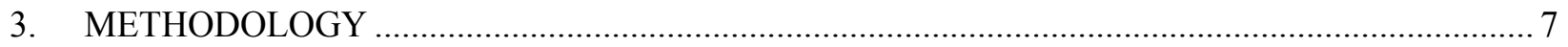

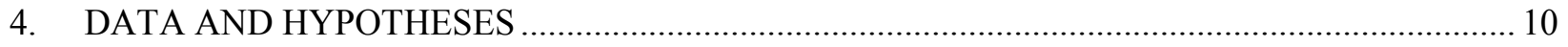

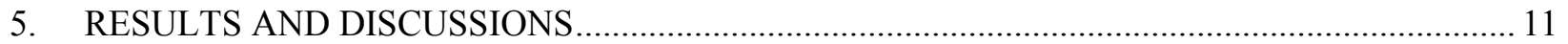

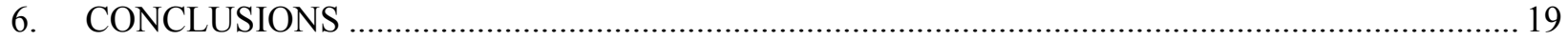

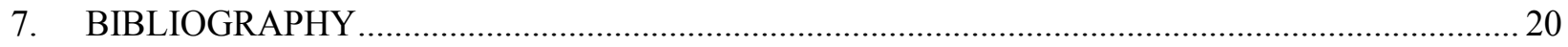




\section{LIST OF FIGURES}

Figure 1: Correlation between traffic flow and information centrality (Sioux-Falls data) ...................... 12

Figure 2: Normal P-P plot-flow vs. information centrality (Sioux-Falls data) ....................................... 13

Figure 3: Correlation between traffic flow and closeness centrality (Sioux-Falls data) .......................... 13

Figure 4: Normal P-P plot-flow vs. closeness centrality (Sioux-Falls data) .......................................... 14

Figure 5: Correlation between traffic flow and betweenness centrality (Sioux-Falls data)...................... 14

Figure 6: Normal P-P plot-flow vs. betweenness centrality (Sioux-Falls data)...................................... 15

Figure 7 Correlation between Flow and Closeness Centrality (Chicago data) ....................................... 16

Figure 8 Normal P-P plot-flow vs. closeness centrality (Chicago data) ................................................... 16

Figure 9 Correlation between flow and betweenness centrality (Chicago data) ....................................... 17

Figure 10 Normal P-P plot--flow vs. betweenness centrality (Chicago data) ........................................... 17

Figure 11 Accessibility (Number of zones affected along Y-Axis) vs. Edge removal (Along X-

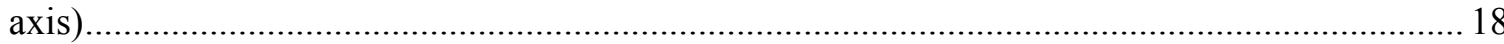

Figure 12 Total System Travel time (along Y-Axis) vs. Edge removal (along X-Axis) curve. ................. 18 


\section{LIST OF TABLES}

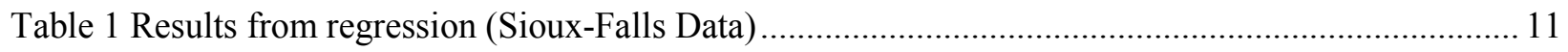

Table 2: Estimated results from regression (Chicago data)..... 


\begin{abstract}
Surface transportation road networks share structural properties similar to other complex networks (e.g., social networks, information networks, biological networks, and so on). This research investigates the structural properties of road networks for any possible correlation with the traffic characteristics such as link flows those determined independently. Additionally, we define a criticality index for the links of the road network that identifies the relative importance in the network. We tested our hypotheses with two sample road networks. Results show that, correlation exists between the link flows and centrality measures of a link of the road (dual graph approach is followed) and the criticality index is found to be effective for one test network to identify the vulnerable nodes.
\end{abstract}

\title{
1. INTRODUCTION AND DEFINITION OF THE PROBLEM
}

Transportation road networks are often recognized as complex due to the topology and traffic dynamics related to it. The economic influence, behavioral processes of the trip makers and uncertainty in the supply-demand characteristics of the network contribute to the complexity of the road transportation networks. Road networks possess structural properties analogous to complex networks like social or communication networks. Most approaches in the transportation system analysis do not account for the structural properties of the road network. Researchers from diverse fields (physical sciences, operation research, economics, etc.) have analyzed transportation systems, but very few have attempted to analyze the system from perspective of network science and explore the structural properties of surface road networks. Among different structural attributes, centrality measures are often of prime interest for the researchers.

In the context social network systems, centrality measures are applied to assess the relative importance of the nodes (i.e., the actors). Further, it is often assumed that correlation exists between structural centrality, and influence and/or power in-group processes (Crucitti, 2006). Centrality measures are related to the network performance that is defined by network connectivity and flow throughput. In case of transportation networks, the traffic flow on each link is most commonly determined by means of nonlinear function of generalized cost of travel. In the current literature, no significant efforts are made to incorporate centrality measures to the computational process of link flows. The way centrality measures of nodes in a network are defined, nodes with higher centrality value are expected to carry higher flow due to their location, and connectivity attributes. This research aims to apply statistical techniques using empirical data to investigate the possible correlation between traffic flow and centrality measures.

Moreover, centrality measures can help to find the most critical road links analogous to social network analysis. This idea leads to the assessment of vulnerability in surface road networks. Assessing the vulnerability of critical infrastructures is getting more attention in the past few years. Initially, the focus was on the information, telecommunication, and the regime of energy networks. However, when the infrastructure of the nation is viewed as a system of systems it becomes clear that all the critical systems are interconnected with each other and interdependency plays a major role. Failure of a particular system will make other components of the global system vulnerable.

With this perception of vulnerability it is evident that road transportation networks have interdependence with communication and energy networks. Therefore, we need to know the possible causes and also the patterns of road network vulnerability for a robust infrastructure management system. This work defines a system to be vulnerable if the functionality of the system can be challenged (reduced or compromised) through intentional or unintentional perturbations in its components. This general definition would put almost all systems in the vulnerable group. In the context of road transportation networks, by assessing vulnerability we mean to identify the critical components of a network, those if failed will cause reduced functionality or no functionality at all for the road network system. A natural 
extension is to define the criticality index of the links in a road network. Criticality index is expected to identify the most critical links of a network.

In the following section, a few recent research works will be discussed. Further, we will explain the basic framework to assess vulnerability from the perspectives of complexity and transportation science. Then, two simple road networks (Sioux Falls and Chicago network) will be examined and the definition of criticality index will be applied to assess vulnerability. Finally, we will discuss the results and further research directions.

\section{RELATED WORK}

Assessing the vulnerability of road networks is not a new research direction. Ball et al. (1989) and Corley and Sha (1982) attempted to identify the most vital arcs in a network using topological properties.

Researchers from the network science in most cases emphasize on the centrality measures to assess the critical components (either arcs or nodes) in a network. Crucitti et al. (2006) explore different kinds of centrality measures for networks of urban streets. They considered primal graphs and determined multiple centrality measures related to the efficiency of the network. Although the research is not directly related to assessing vulnerability, it explains the relation between network functionality and centrality measures that can be used to identify the critical components in road networks. Latora and Marchiori (2007) introduced Delta centralities in context of information centrality in a network that can be applied to assess the impact of removing an edge from the network. The analogy is to remove a particular street from transportation road networks. Ukkusuri and Holguin -Veras (2008) used economic welfare concept and centrality measures to identify the critical components in the transportation networks. In their research, an optimization model is developed to find how important a component is in a network under outside attack. When an adversary is interested to attack a vulnerable network, that individual is expected to maximize the disruption to the network which can be achieved by attacking the most critical components. Therefore, the optimization model will effectively identify the most critical link of the road network. Issacharoff $e t$ al. (2009) combined the concepts of transportation science and network statistics to assess the vulnerability of highway road networks. They conducted topological analysis of the graphs representing road networks along with determination of steady state traffic flow through Wardropian-Nash equilibrium search.

As described above, limited efforts are made to solve the problem incorporating all the interacting agents in road transportation networks. The possible correlation between the structural properties (centrality measures) and traffic characteristics (flow) of road links in a transportation networks is not investigated rigorously. Therefore, the main contribution of this research is the exploration of structural properties of road networks for possible correlation with traffic flows on the link. Additionally, the traffic flow dynamics and topological attributes will be considered through Delta centrality measures to assess the critical edge in a road network.

\section{METHODOLOGY}

This section defines the criticality index and describes the algorithm that determines flow on each link of the road network.

\section{Criticality index}

This research puts focus on surface road transportation networks that possess different characteristics compared with social networks. Geographical attributes are one of the most distinct properties of road network. In road networks, the nodes and edges occupy real and physical positions in two dimensional Euclidian spaces and the edges imply physical connections. However due to similarity of topological and structural properties of road network with social network, we can still apply the basic formulations to obtain different structural centrality measures for any node in a road network. In this research we use structural centrality measures of nodes to define the criticality index for any node in the network. 
Centrality measures are commonly defined and obtained for nodes in a network. However we have our interests on the centrality measures of road links or edges in the graph. To resolve this problem, we follow the space syntax (see Hillier and Hanson, 1980) or the dual approach to represent the network. In this approach, the streets are the nodes and the intersections are the edges in a network. The dual of a graph is often mentioned as the Line Graph of the original or primal graph. For any graph $G(N, A)$ we denote the Line Graph as $L(G)$, where $N$ and $A$ represent the sets of nodes and edges respectively of the network. If $u$ and $v$ are two edges in the primal graph (and the nodes in the line graph), there will be connectivity between these two nodes in the line graph if $u$ and $v$ are adjacent in the primal graph. In our research dual graph modeling approach is applied to get a graph in which the nodes are the road links and the edges represent intersections and a centrality based criticality index is computed for all the nodes in the line graph.

Centrality measures can be defined in different manners. The standard centrality measures are based on two distinctive basic ideas: how a node is near to all other nodes in the network (e.g., degree, closeness centrality), and the most central nodes are most likely stand between other nodes (e.g. betweenness, flow betweenness centrality) playing the role of intermediary. Latora and Marchiori (2007) introduced delta centrality measures for networks which is a combination of the two concepts mentioned above.

Information centrality is a special instance of the family of delta centrality that accommodates the flow efficiency of a network. Since connectivity and commodity flow (human travelers and goods) are two key components while assessing the importance of a road network, we attempt to formulate the criticality index of nodes based on information centrality. Information centrality of any node can be defined as the relative drop in the efficiency of the network when that node is removed or deactivated (see Latora and Marchiori, 2007). Information centrality can mathematically be expressed as:

$$
C_{i}^{I n}=\frac{\Delta P}{P}=\frac{\Gamma(G)-\Gamma\left(G^{*}\right)}{\Gamma(G)}
$$

Here, $\frac{\Delta P}{P}$ measures the change in network performance when the particular node is removed from the network. Further, $\Gamma(G)$ is a measure of network efficiency and can take different forms based on the objective of the study. It can be the number of edges, path length, etc. Geodesics are often used in case of information exchange or flow. Next we define $\Gamma(G)$ in the context of road networks.

According to spatial interaction principles, flow efficiency of the exchange (movement) of goods and people along the road network is inversely proportional to the shortest path distance between the origin destination pairs in a road. Latora and Marchiori (2001) introduced a metric to measure how efficiently nodes in a graph exchange information based on geodesics in an information network. Analogous to this, we can define an efficiency measure for road networks where the shortest path distance between the any origin-destination pair can be approximated as the Euclidian distances between them. Using this measure of efficiency we can determine the information centralities of the nodes in a road network.

Network efficiency can be determined as follows:

$$
\Gamma(G)=\frac{1}{N(N-1)} \sum_{i, j \in G, i \neq j} \frac{d_{i j}^{\text {Eucledian }}}{d_{i j}}
$$

When the information centrality is determined, we can define the criticality index of a node (i.e., road link) as follows:

$$
C_{i}^{V}=\alpha C_{i}^{I n}+\delta_{i}^{Z} \beta \frac{C_{i}^{Q}}{\sum_{i \in N} C_{i}^{Q}}
$$

In this expression,

$$
C_{i}^{V}=\text { criticality index for any node } i
$$


$\alpha, \beta=$ constants representing weights to flow and demand respectively (here both values are 1 )

$\delta_{i}^{Z}=1$, when the node is a center of trip attraction zones (e.g. a business center, town center where most

trips will be made)

$=0$, otherwise

$C_{i}^{Q}=$ Total demand attracted to zone $i$ (trip rates)

Unlike information or network science, the trip demand and flows on the link are totally different quantity in context of transportation science. Using this criticality index we rank the nodes and higher value of criticality index indicates the node is more critical.

\section{Traffic flow using stochastic user equilibrium}

Stochastic user equilibrium (SUE) formulates the network equilibrium model based on random utility theory. In a road network for any origin $r$ and destination $s$, let $T_{k}^{r s}$ be the perceived travel time for any path $k$ and $t_{k}^{r s}$ is the actual measured travel time. Now the relation can be expressed as, $T_{k}^{r s}=t_{k}^{r s}+\chi_{k}^{r s} \quad, \forall k, r, s$

Here, $\chi_{k}^{r s}$ is the random error term specific to the route (captures all the unobserved factors associated with the choice making). Again, it is also assumed that, $E\left[\chi_{k}^{r s}\right]=0$, i.e. the average perceived travel time will be the same as measured travel time. Now, the share of drivers choosing the $k$-th route can be expressed as the probability of choosing $k$-th route over any other $l$-th route.

$P_{k}^{r s}=\operatorname{Pr}\left(T_{k}^{r s} \leq T_{l}^{r s}\right), \forall l \in K_{r s}$; Where $K_{r s}$ is the set of all routes connecting $r$ and $s$ origin-destination pairs.

The probability that a given route is chosen is simply the probability that its travel time is perceived to be the lowest of all the alternative routes.

Now, we assume the error terms associated with the routes are independent and identically distributed and follows Generalized extreme value type-I distribution. Therefore, the probability expression can be written as:

$$
P_{k}^{r s}=\frac{e^{-\theta t_{k}^{r s}}}{\sum_{l} e^{-\theta t_{l}^{r s}}}, \forall k, r, s
$$

Here, $\theta$ is a constant that scales the perceived travel time sensitivity.

When the probabilities are computed we can load the network. The mechanism of assigning traffic volumes to the road links of a network is known as network loading process in the context of traffic assignment which is the last step of traditional trip based four step transportation planning process. One of the network loading models regarding SUE is the STOCH networking model. STOCH is a route choice model based on multinomial logit formulation. STOCH is also known as Dial's algorithm. The number of paths between any origin and destination in a realistic network is extremely large and the path enumeration takes a lot of time in terms of computation. So it is necessary to use intelligent algorithms to avoid the path computations in the process of network loading. The special characteristic of the STOCH algorithm is : we do not need to enumerate all the paths to assign the flows based on generalized travel cost. The algorithm identifies a route between any specific origin destination pair to be reasonable only if the route includes links that take the traveler further away from the origin and closer to the destination.

The initial STOCH algorithm contains two basic modules: forward pass (link weights) and backward pass (assigning flows). However, it requires two minimum path calculations (shortest path) for each origin destination pair in the network. This makes the algorithm computationally almost infeasible in the context of large networks. To resolve this problem, we can modify the definition of reasonable paths in the network that can effectively exploit the one-to-all shortest path algorithm as described in Dijkstra's shortest path algorithm. The new definition is : 


\section{A path would be considered reasonable if it includes only links that do not take the traveler back toward the origin.}

Now, the complexity of the problem is much more less than the earlier one. The modified algorithm is known as the Single-Pass STOCH algorithm and there is no empirical evidence of more accuracy of the original STOCH (double pass) algorithm over the Single Pass STOCH. In the next section we will describe the steps in the algorithm and its different modules.

\section{DATA AND HYPOTHESES}

We compute and apply criticality measures on two test networks. will be investigated in the current research. Both of them represent transportation road network and the traffic flows on the links will be computed using the deterministic traffic user equilibrium formulation.

\section{Sioux-Falls network:}

Sioux Falls is the largest city in the U.S. state of South Dakota. It is considered as the fastest growing metro in the Midwest with a total growth of $22 \%$ in $2000^{1}$. We use the road network of the city of Sioux Falls as a test bed for the proposed formulation. Note that, Sioux Falls network is often considered as $\operatorname{artificial}^{2}$ because of its slight contrast with the actual network. The network consists of 24 nodes and 76 arcs. For traffic flow analysis, 24 zones will be considered. As per the four- step transportation planning process trips are originated in and attracted to the zones. Thus, there will 24 origin destination pairs and the trip demands (as a trip-table) are provided with the data. In the transportation science literature, variations of Sioux Falls network exist, but the variations are mostly in the generalized cost ${ }^{3}$ values.

\section{Chicago sketch network:}

This data set represents an aggregated sketch of the Chicago region and the data set is provided by the Chicago Area Transportation Study. The trip table (i.e., the demand values) mostly represents low-tomedium congestion scenario in the region. Past studies suggest using higher values of trip demand to make the representation more realistic while assessing the effectiveness of any algorithm. The network has 933 nodes and 2950 links. There are 387 zones in the network.

\section{Hypothesis 1:}

The traffic flow values are correlated with the structural properties (centrality values) of the links (nodes in the dual graph). The links with higher centrality values are expected to carry more flows compared to links with smaller centrality values.

To test this hypothesis, we compute different centrality measures for the nodes in the dual graph. The centrality measures include: closeness, betweenness, flow betweenness, and information centrality. We used the tool UCINET for this purpose. Further, using the STOCH algorithm (described in the earlier section) the link flow values are computed. We developed a program in visual $\mathrm{C}++$ that gives the link flows as output. To find any correlation that exists between flow values and centrality measures, a simple linear relationship is assumed between them. We consider the link flow as dependent variable and centrality measures as the explanatory variables. Using simple regression the coefficient values and corresponding t-stat values are examined to reach any conclusion about the hypothesized correlation. Again, we plot the centrality measures and flow values on the same graph to investigate the data fit (R-Squared value).

\footnotetext{
${ }^{1}$ http://en.wikipedia.org/wiki/Sioux Falls, South_Dakota

${ }^{2} \mathrm{http}: / /$ www.bgu.ac.il/ bargera/tntp/

${ }^{3}$ Travel cost from node $\mathrm{i}$ to $\mathrm{j}$ can be expressed as a generalized cost comprising travel time, tolls, perceptions, latent choices etc. Here we are assuming the generalized cost function is given and the formulation of the function is beyond the scope of this research paper.
} 


\section{Hypothesis 2:}

\section{The most critical nodes in the network have higher value of criticality index.}

Two key performance measures: total system wide travel time and accessibility will be investigated to validate this hypothesis. Using function defined in the algorithm section, we compute the criticality indices of the nodes and ranked according to their values. The higher values indicate more importance for the node (i.e. the road link in the network). Then a distinct approach is adopted to investigate the efficacy of the criticality index values. First the edges in the network are removed in a random fashion and the network performance is computed after each edge removal. Then, the edges are removed again but this time according to centrality index values and performance measures are computed. We plot them in the same graph to observe the deviation pattern. When the edges are removed using criticality index, we would expect a sharper drop of performance compared to random edge removal.

\section{RESULTS AND DISCUSSIONS}

\section{Results for Hypothesis 1:}

The following table presents the results obtained from linear regression models in which link flow is dependent variable and centrality measures are the regressors. Both link flow and centrality measures are normalized and are unit less.

\section{Results for Sioux Fall Data:}

Table 1 Results from regression (Sioux-Falls Data)

\begin{tabular}{|l|l|l|l|}
\hline Models/Independent variables & Coefficient & t-stat & Prob \\
\hline Dependent Variable & & & \\
\hline Link flow & & & \\
\hline Explanatory Variables & & & \\
\hline Model-1 & & -1.032 & 0.309 \\
\hline Constant & -0.259 & 3.036 & 0.004 \\
\hline CloseNess Centrality & 0.947 & & \\
\hline & & & 0 \\
\hline Model-2 & & 5.208 & 0.002 \\
\hline Constant & 0.32 & 3.299 & \\
\hline BetweenNess Centrality & 0.492 & & \\
\hline Model-3 & & & 0.84 \\
\hline Constant & & -0.203 & \\
\hline Information Centrality & -0.042 & 2.622 & 0.13 \\
\hline & 0.685 & & \\
\hline Model-4 & & & 0.562 \\
\hline Constant & 0.127 & 0.586 & \\
\hline
\end{tabular}




\begin{tabular}{|l|l|l|l|}
\hline Models/Independent variables & $\underline{\text { Coefficient }}$ & $\underline{\underline{\text { t-stat }}}$ & $\underline{\underline{\text { Prob }}}$ \\
\hline BetweenNess Centrality & 0.386 & $\underline{2.057}$ & 0.047 \\
\hline Information Centrality & 0.294 & $\underline{0.936}$ & 0.355 \\
\hline Model-5 & & & \\
\hline Constant & & & \\
\hline BetweenNess Centrality & -0.004 & -0.012 & 0.991 \\
\hline Information Centrality & 0.33 & $\underline{1.536}$ & 0.134 \\
\hline CloseNess Centrality & -0.042 & $\underline{-0.061}$ & 0.951 \\
\hline
\end{tabular}

First, we include individual centrality measures in the regression (Model 1-3). From Model-1 and Model2 we can conclude that, closeness centrality and betweenness centrality are linearly correlated with flows on the links. The $t$-stat values on the coefficients of closeness and betweenness centrality imply that with $95 \%{ }^{4}$ confidence the coefficients are different from zero and thus statistically significant. However, model-3 indicates the information centrality is not linearly correlated with flow values if the t-stat value of the coefficient on information centrality is considered.

Further, we included different centrality measures at the same time as explanatory variables (in model-4 and model-5). But, the coefficients are statistically insignificant in both of the cases as shown in the table above. In following figures the plots with respective $\mathrm{R}^{2}$ values are shown:

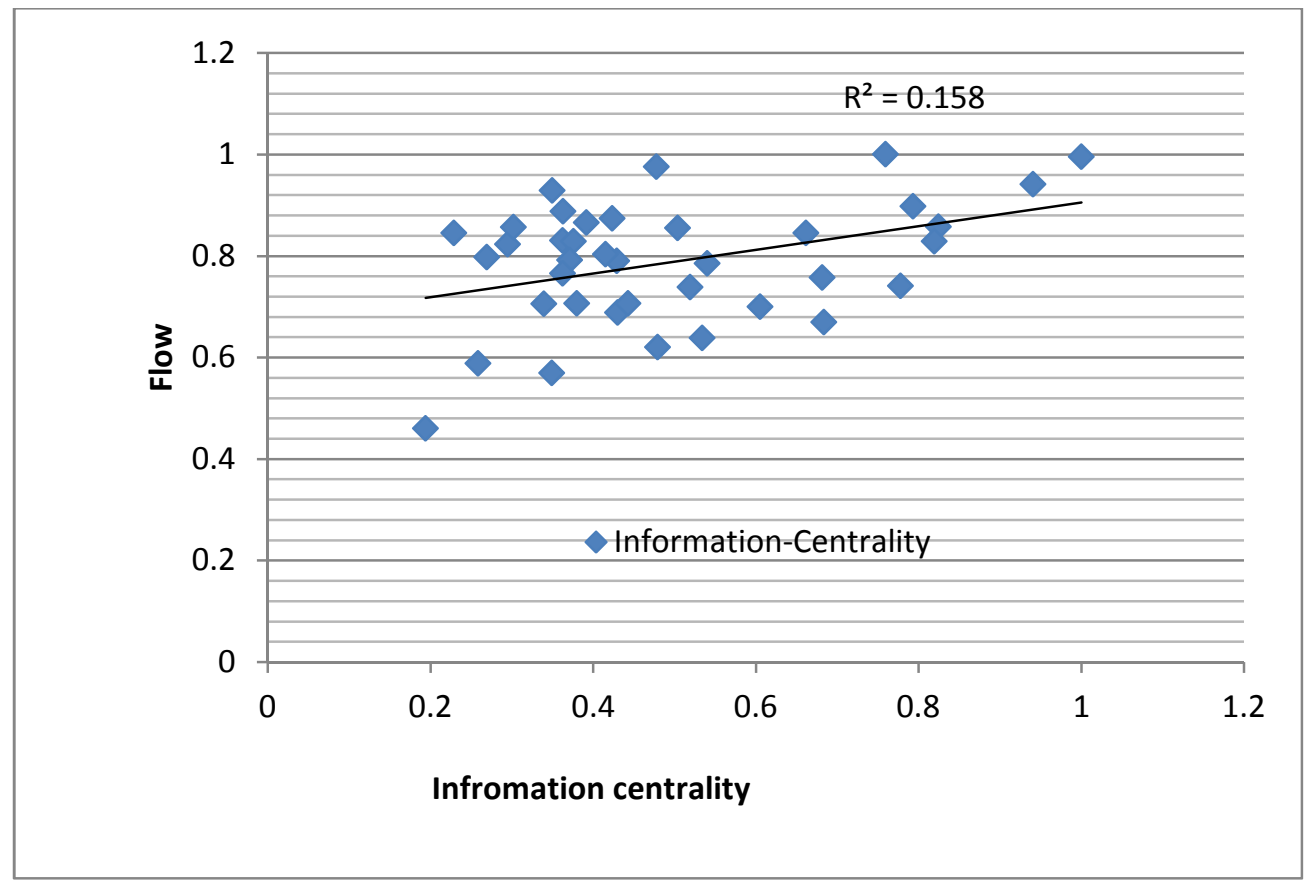

Figure 1: Correlation between traffic flow and information centrality (Sioux-Falls data)

\footnotetext{
${ }^{4} 95 \%$ confidence is only an arbitrary choice to indicate significance, in this research we use $95 \%$ for more accuracy
} 


\section{Normal P-P Plot of Regression Standardized Residual}

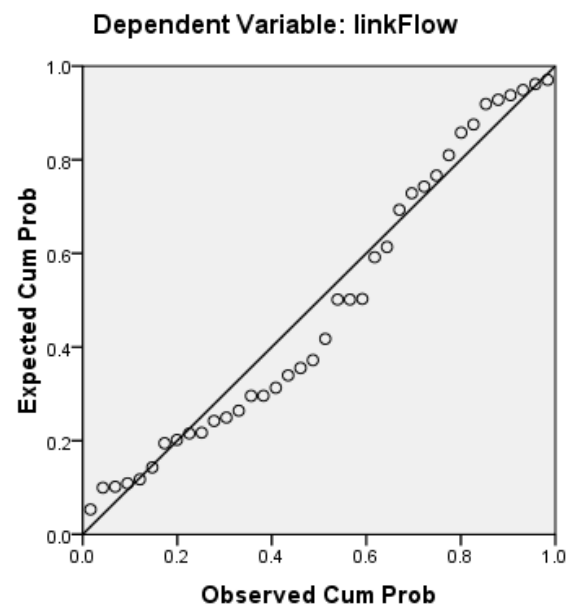

Figure 2: Normal P-P plot-flow vs. information centrality (Sioux-Falls data)

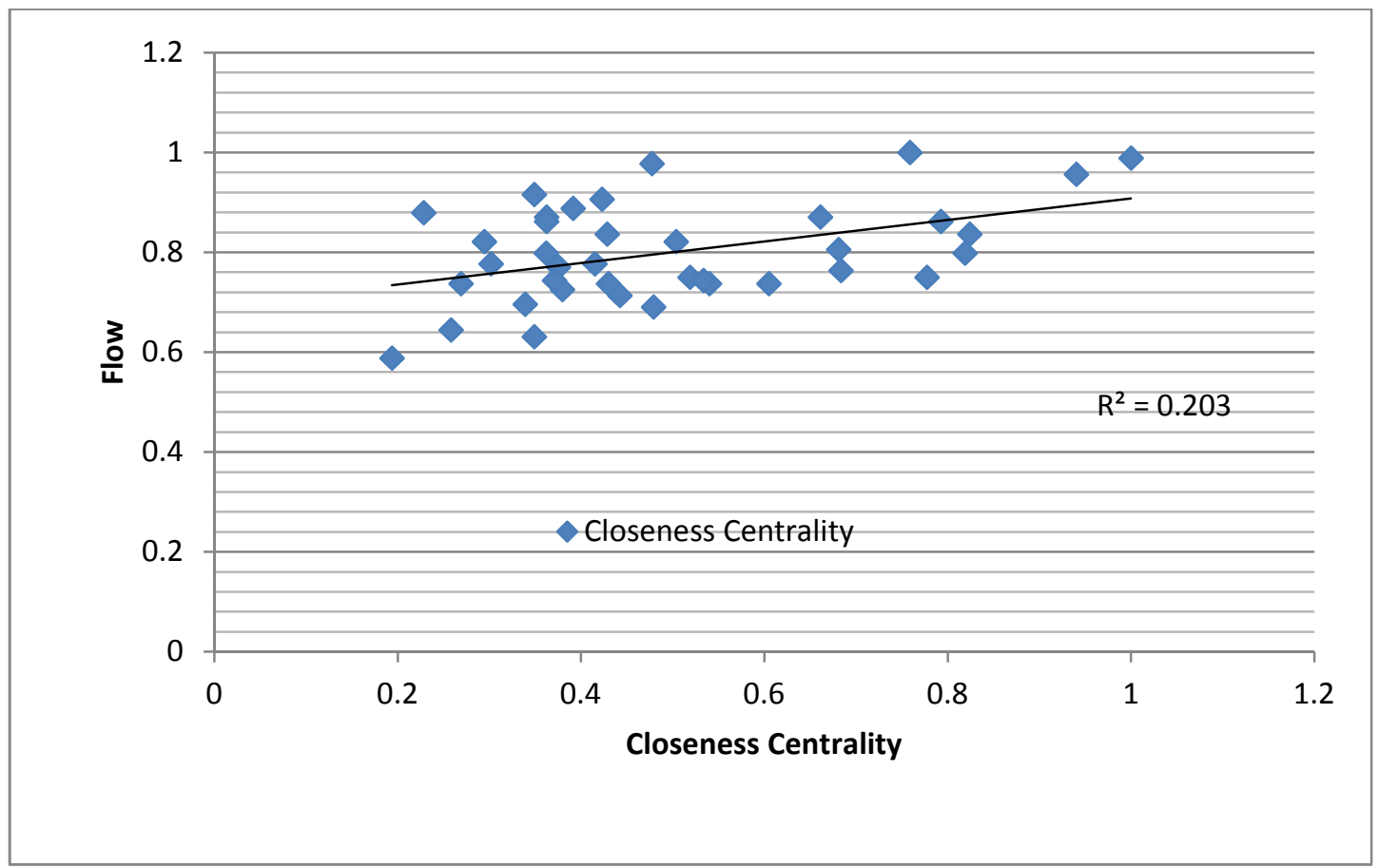

Figure 3: Correlation between traffic flow and closeness centrality (Sioux-Falls data) 
Normal P-P Plot of Regression Standardized Residual

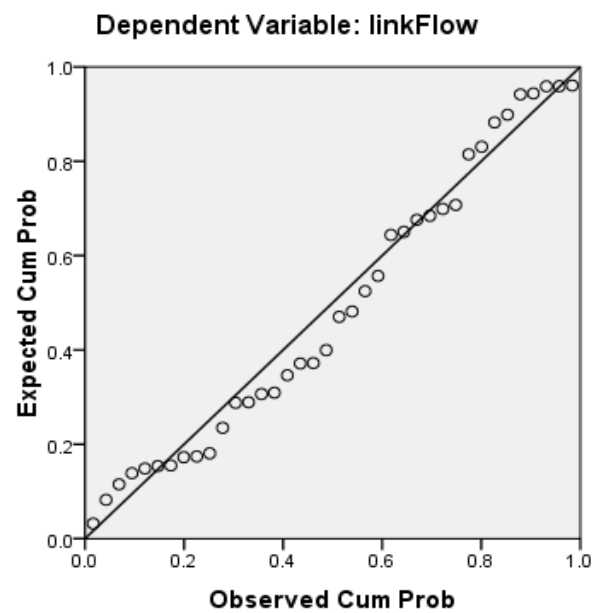

Figure 4: Normal P-P plot-flow vs. closeness centrality (Sioux-Falls data)

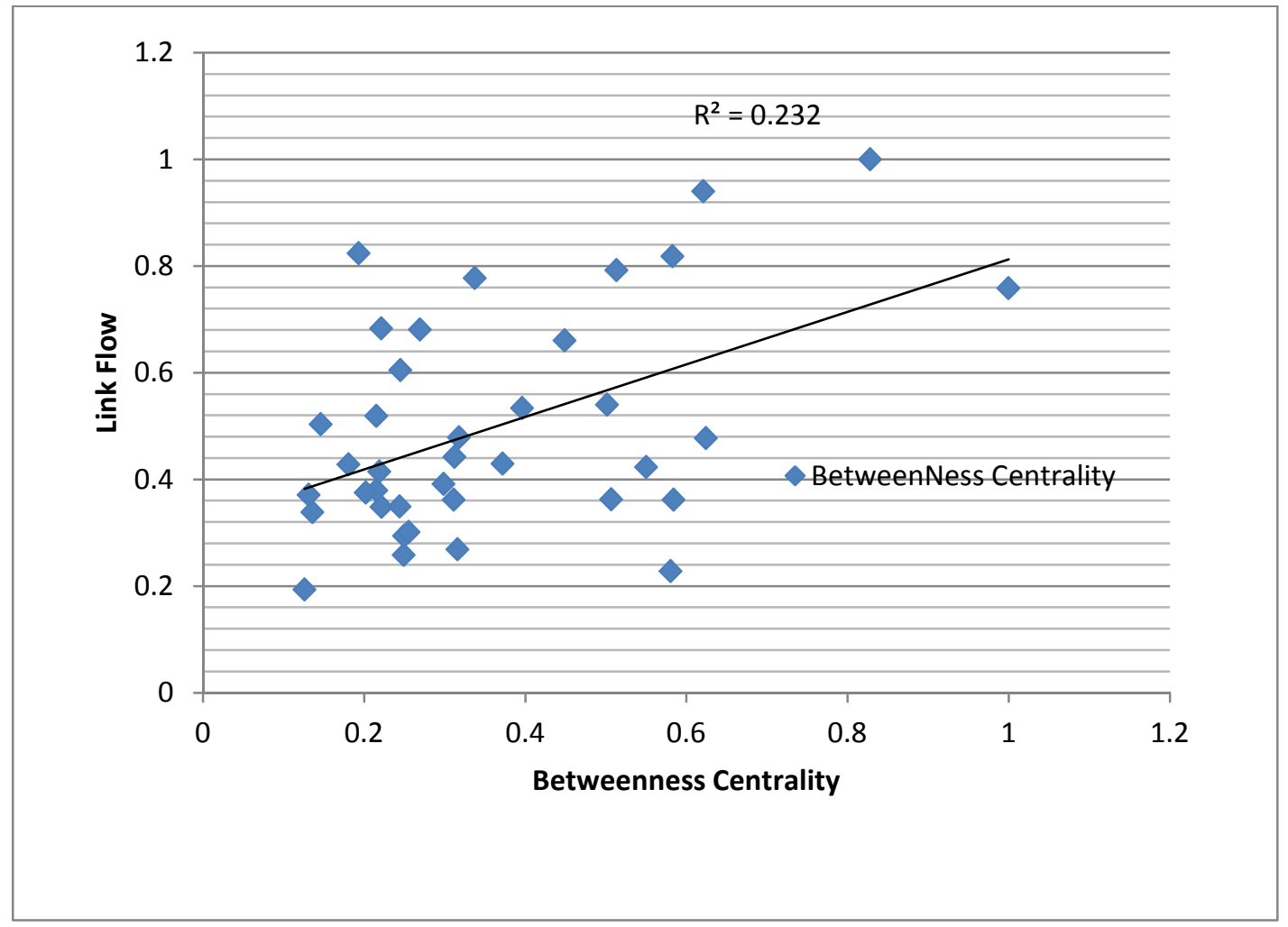

Figure 5: Correlation between traffic flow and betweenness centrality (Sioux-Falls data) 


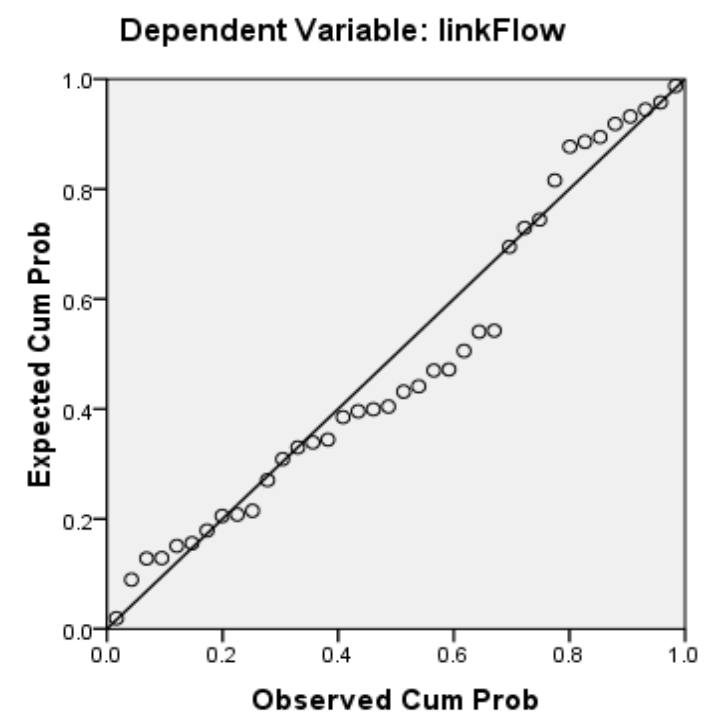

Figure 6: Normal P-P plot-flow vs. betweenness centrality (Sioux-Falls data)

\section{$\underline{\text { Results for Chicago data: }}$}

Table 2: Estimated results from regression (Chicago data)

\begin{tabular}{|l|l|l|l|}
\hline Models/Varaibles & Coefficient & $\underline{\text { t-stat }}$ & $\underline{\text { Prob }}$ \\
\hline Dependent Variable & & & \\
\hline Link flow & & & \\
\hline & & & \\
\hline Model-1 & & & \\
\hline Constant & -7.534772664 & -7.82097 & 0.00000 \\
\hline CloseNess Centrality & 8.097182868 & 8.146282 & 0.00000 \\
\hline & & & \\
\hline Model-2 & & & \\
\hline Constant & 0.141943101 & 4.902536 & 0 \\
\hline BetweenNess Centrality & 0.653010631 & 6.792568 & 0 \\
\hline
\end{tabular}

The coefficients have high t-stat values for closeness and betweenness centrality. One should notice that the constant in each regression also has a very high value. This implies that, there are other unobserved factors that affect link flows. 


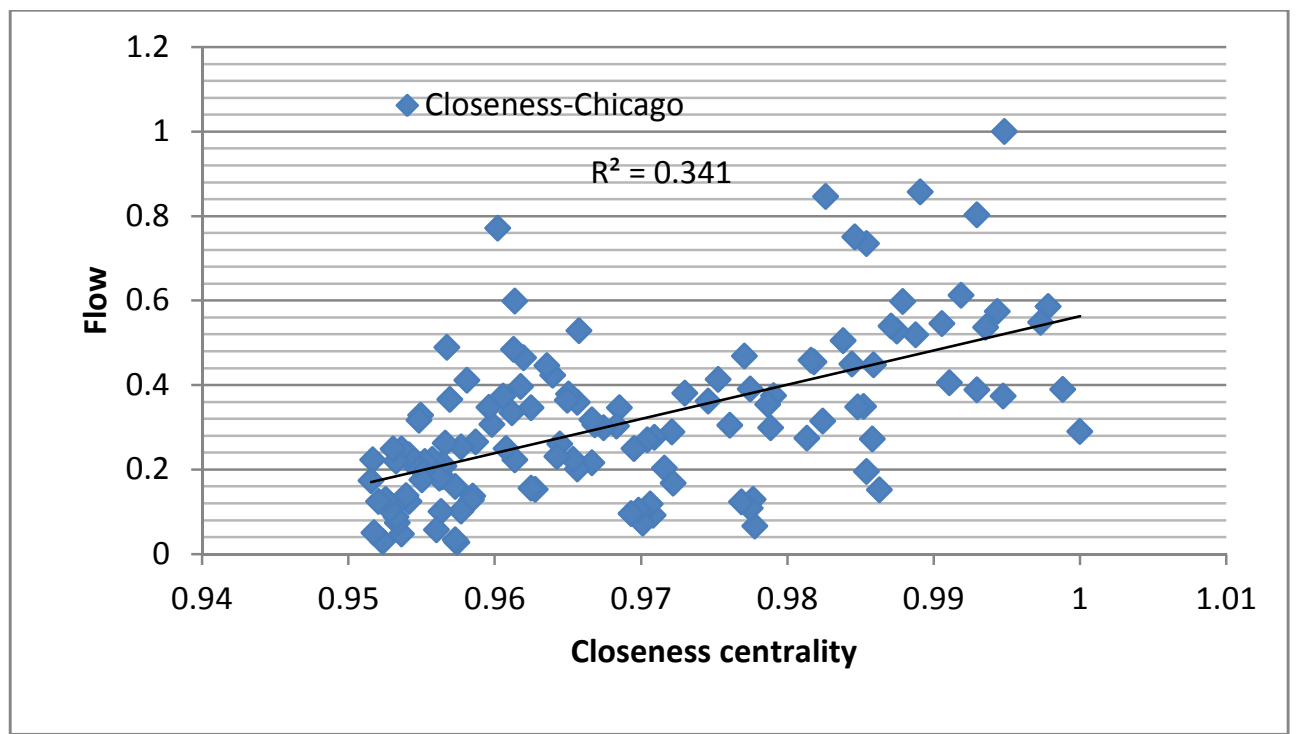

Figure 7 Correlation between Flow and Closeness Centrality (Chicago data)

\section{Normal P-P Plot of Regression Standardized Residual}

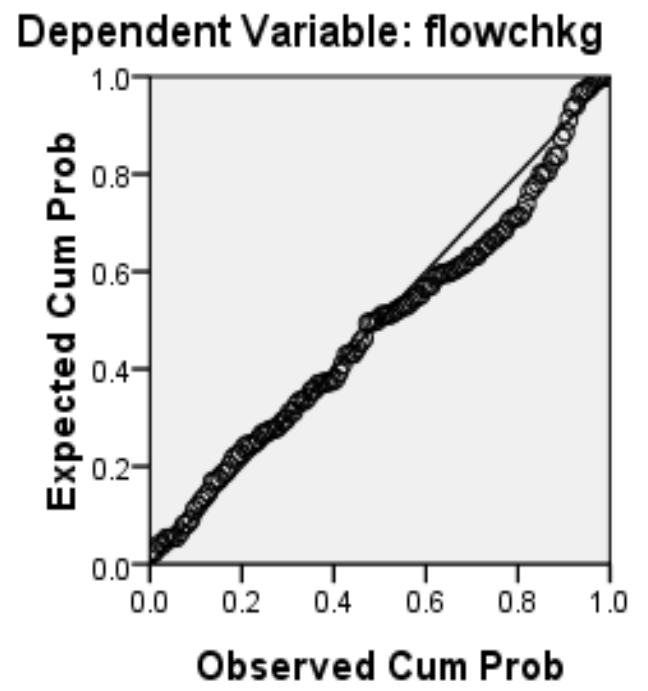

Figure 8 Normal P-P plot-flow vs. closeness centrality (Chicago data) 


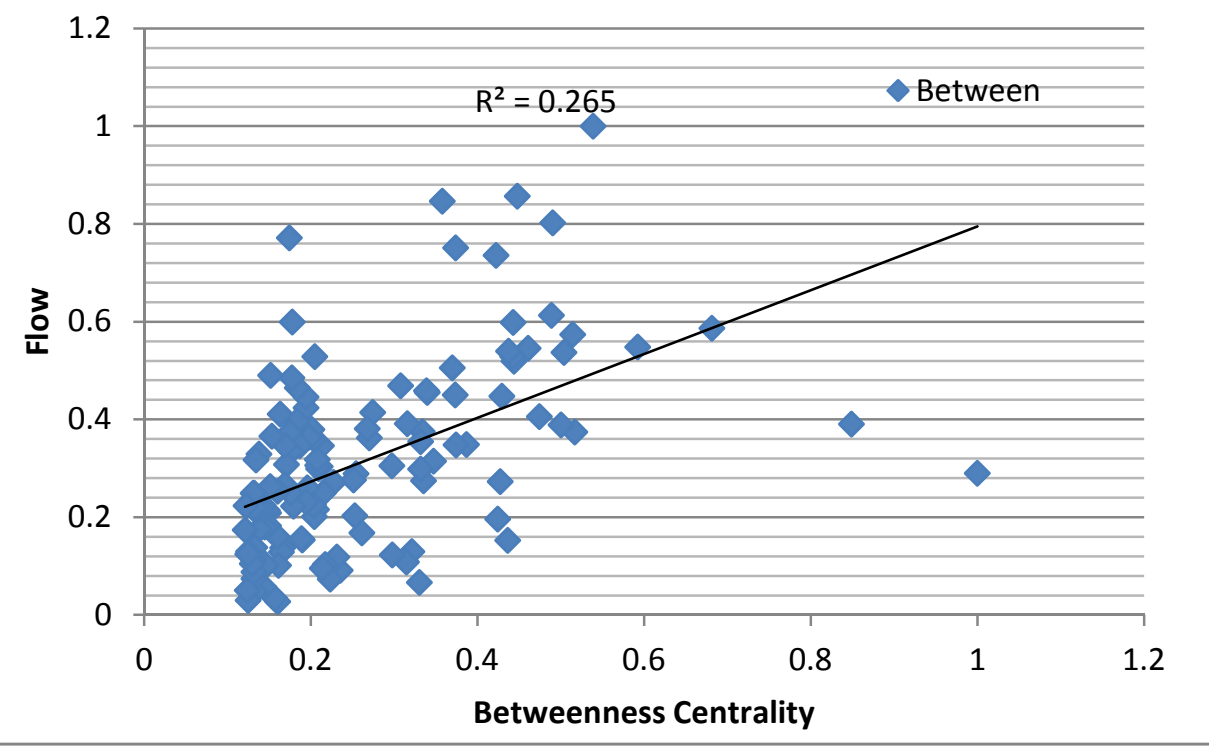

Figure 9 Correlation between flow and betweenness centrality (Chicago data)

Normal P-P Plot of Regression Standardized Residual

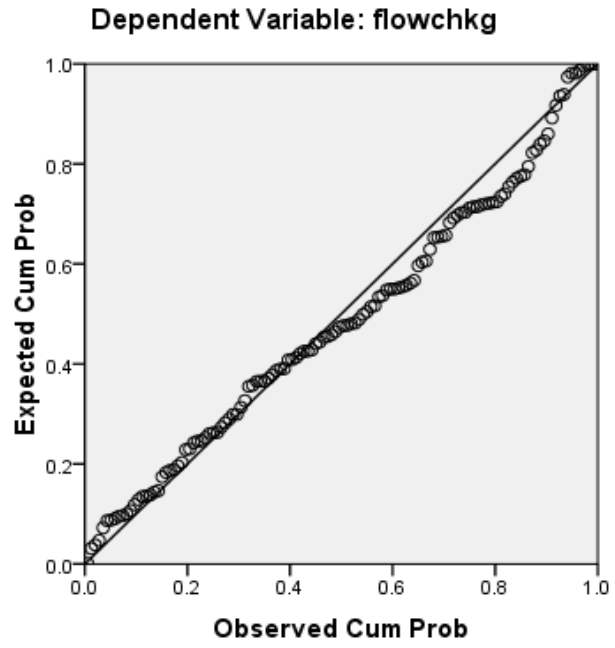

Figure 10 Normal P-P plot--flow vs. betweenness centrality (Chicago data) 


\section{Results for Hypothesis 2:}

The following graphs are plotted for Sioux-Falls data. Results for Criticality Index effectiveness:

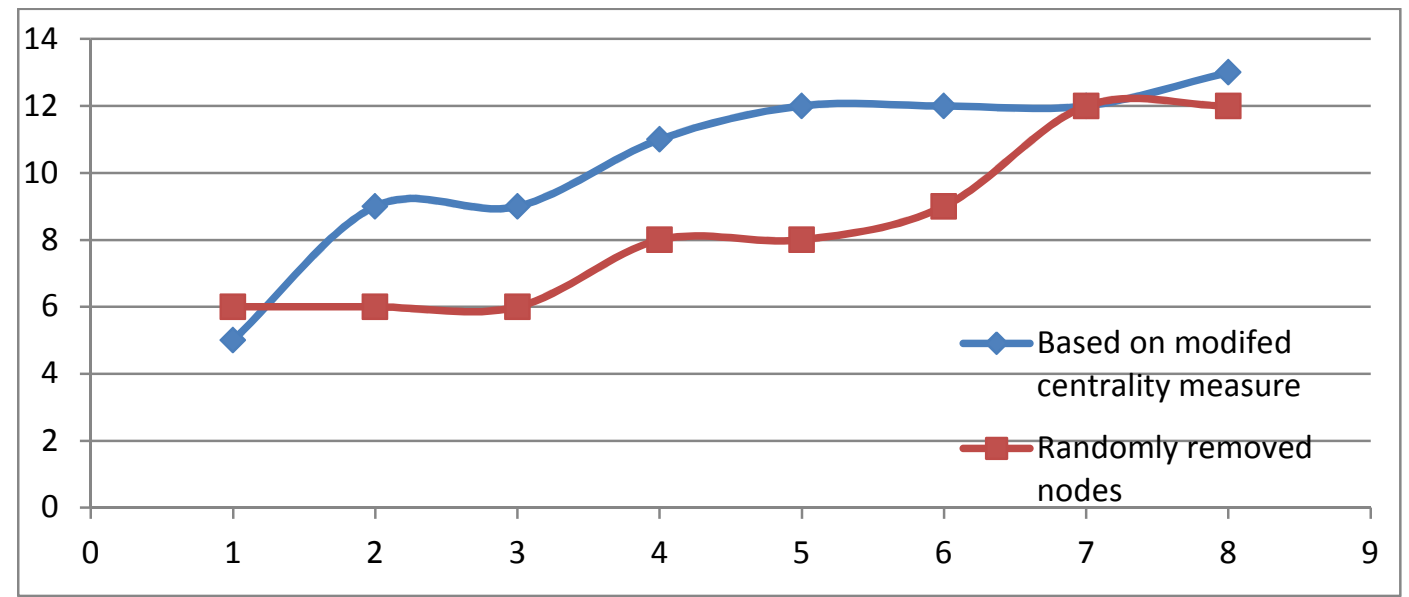

Figure 11 Accessibility (Number of zones affected along Y-Axis) vs. Edge removal (Along X-axis)

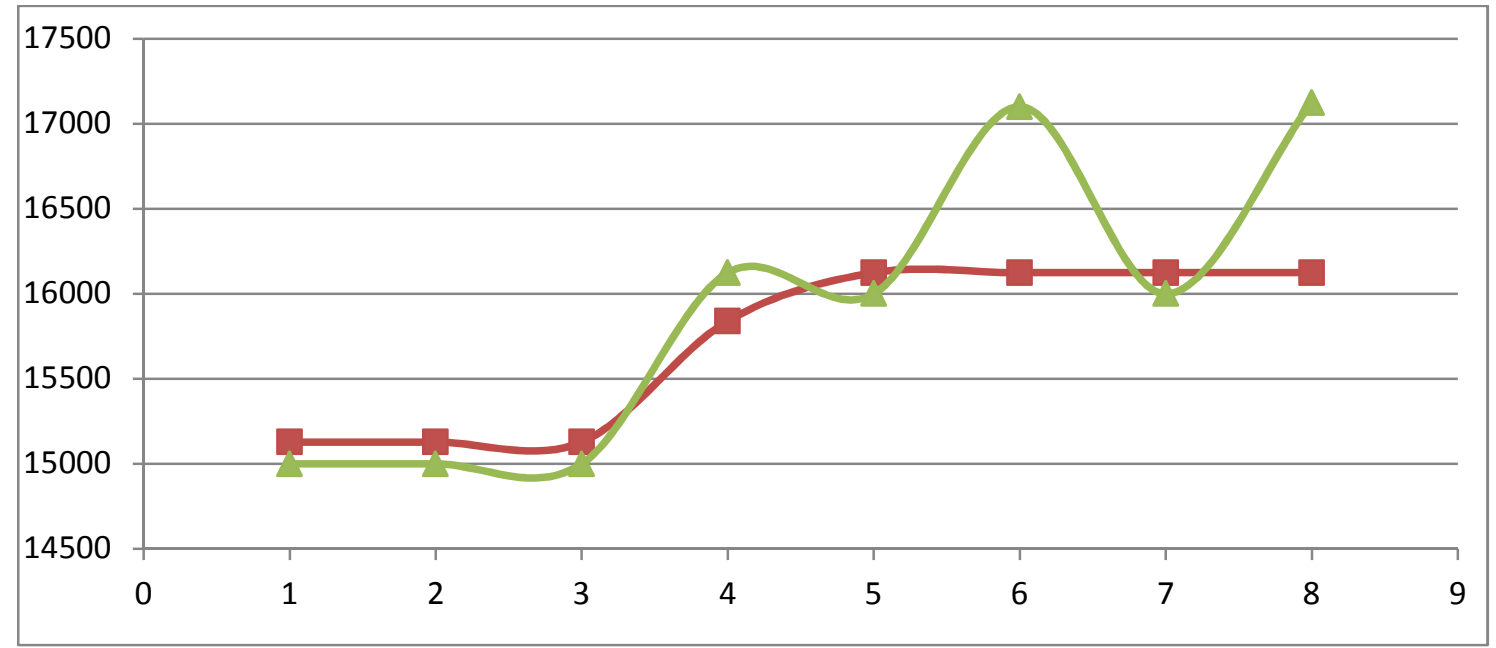

Figure 12 Total System Travel time (along Y-Axis) vs. Edge removal (along X-Axis) curve.

In the first graph (Fig. 5.3) we can observe the effect of edge removal based on criticality index is more compared to the case when the edges are removed randomly.

For the second graph (Fig. 5.4), initially the increment of total system wide travel time is more (increment in travel time indicates reduction in network performance), but later the effect stabilizes. The effect of removal of random edges is not uniform rather random in manner.

\section{Results for Chicago Data:}

In this network there are 387 by 386 origin destination pairs. Since the network is large, there are many alternative routes and removing just a few edges (5-10) do not affect the total system travel time or accessibility to a significant level. For example, when we remove 5 edges the total system travel time 
reduction is expected to be $15 \sim 18$ minutes. Since the total system travel time and accessibility do not show much deviation, we do not include them in our results.

\section{Correlation between flow and centrality measures:}

- The $\mathrm{R}^{2}$ values are very low in plotted graphs for both Sioux Fall and Chicago Data. However, $\mathrm{R}^{2}$ value only indicates how much variation is explained by the available data. This implies, there are other variables those contribute to the link flow values in the road network. This makes sense because flows on the link depends on many factors ( e.g. travel time, route choice, weather, personal characteristics like income, vehicle ownership). Additionally, as mentioned in the algorithm section the link flows are computed from a probabilistic logit based model that accounts for unobserved factors. So, it is reasonable to get low values when we are using a single explanatory variable.

- The most important thing to notice is that, the coefficients on the centrality measures (specifically for closeness and betweenness centrality) are statistically significant (95\% level). This indicates the presence of correlation between link flow and structural centrality measures.

- So, the results obtained from our two specific data sets support the first hypothesis.

\section{Effectiveness of criticality index:}

The plotted graphs (see Fig. 5.3 and Fig. 5.4) clearly indicate drop in system performance when edges in the road network are removed based on the criticality index values. When we compare (Sioux Fall data) this with the case where the edges are removed in a random fashion, it is observed that the deviation takes place relatively faster in case where we use centrality index values to remove the edges. However the deviation in system performance for large network like Chicago sketch is found to be very small and not significant. 387 by 386 or 149382 origin destination pairs are computationally expensive when we have to consider each origindestination pair separately to assign flows and compute the system travel time. Additionally for each origin-destination pair, when we remove the edges a new configuration of the network occurs and added to the complexity of the problem. For these reasons, the Chicago network is examined only for few zones and the results were not significant.

If we consider the results obtained from Sioux Fall network, then we can conclude that the criticality index values are effective to identify the critical edges of the network that affect the system performance of the network and our second hypothesis is supported for Sioux Fall network. However, we need to verify it for other data sets to reach a generalized conclusion.

\section{CONCLUSIONS}

This research investigates the structural graph properties of surface road transportation networks. Surface transportation road networks have the topological properties similar to complex social networks, but very few attempts are made to explore the structural properties of road networks. In this research, we investigate the correlation between traffic characteristics i.e. link flows and centrality measures. We assumed linear relationship between link flow and the structural centrality values and used simple regression techniques to find correlation. The centrality measures are used as explanatory variables for 
link flows and found to be statistically significant (closeness and betweenness centrality measures) with $95 \%$ confidence interval.

Further, we have defined criticality index to identify the important road links in a network. The graphical plots (Edge removal vs. efficiency drop) clearly show the effectiveness of the index. However the effectiveness could not be shown for the larger Chicago network. The most important finding of this research is the correlation between traffic characteristics and structural characteristics of surface road transportation networks. Since betweenness centrality measures capture the importance of the nodes when the network is meant for flow of some entity (information or commodity or human or else) intuitively we can say it is feasible to find correlation between flow and centrality measures and it is also observed in the results we have shown here. Additionally, the criticality index can serve as useful direction for assessing vulnerability. However, we need to validate its effectiveness with larger data sets.

We followed a very simple statistical method is this research to show correlation. This is specific to the road networks we used only. Again, correlation does not mean causality. The correlation may be just the artifact of data sets only.

As a future research direction, both structural properties (centrality measures) and traditional traffic characteristics can be jointly formulated in a single function to explain the link flows. Additionally, the economic importance can also be included for trip attraction zones and we can modify the criticality index to identify critical components of a network comprising different infrastructures instead of roads.

\section{BIBLIOGRAPHY}

1. Ahuja, Ravindra K., Thomas L. Magnanti, and James Orlin. Network Flows:Theory, Algorithms, and Applications. Prentice Hall, 1993.

2. Ball MO, Golden BL, Vohra RV (1989).Finding the most vital arcs in a network. Oper. Res. Letter, 8:73-76.

3. Corley, H., Sha DY (1982). Most vital links and nodes in weighted networks. Oper. Res. Letter; 1:157-360.

4. Crucitti, P., Vito Latora, and Sergio Porta.(2006). Centrality in networks of urban streets. Physics. (DOI: 10.1063/1.2150162)

5. Hillier B. and Hanson J. (1984). The Social Logic of Space. Cambridge University Press: Cambridge.

6. Issacharoff, L., Lämmer, S., Rosato, V., Helbing, D., 2008. Critical Infrastructures Vulnerability: The Highway Networks, in: Helbing, D. (Ed.), Managing Complexity:

Insights, Concepts, Applications. Springer Berlin Heidelberg, pp. 201-216.

7. Latora, V. and M Marchiori.(2007). A measure of Centrality based on network efficiency. Journal of Physics.

8. Sheffi,Y. (1983). Urban Transportation Networks: Equilibrium Analysis with Mathematical Programming Methods. Prentice-Hall, Inc. Englewood Cliffs, J 07632.

9. Ukkusuri, Satish V., and Jose Holguin-Veras (2008). Assessing Critical Components in Transportation Systems: Economic models and Complex science Approaches. Transportation Research Record, TRB. 